\title{
Circuit
}

Musiques contemporaines

\section{Les auteurs du numéro}

Volume 13, numéro 1, 2002

L'électroacoustique : à la croisée des chemins?

URI : https://id.erudit.org/iderudit/902268ar

DOI : https://doi.org/10.7202/902268ar

Aller au sommaire du numéro

\section{Éditeur(s)}

Les Presses de l'Université de Montréal

ISSN

1183-1693 (imprimé)

1488-9692 (numérique)

Découvrir la revue

Citer ce document

(2002). Les auteurs du numéro. Circuit, 13(1), 66-67.

https://doi.org/10.7202/902268ar d'utilisation que vous pouvez consulter en ligne.

https://apropos.erudit.org/fr/usagers/politique-dutilisation/ 


\section{LES AUTEURS DU NUMÉRO}

\section{Réjean Beaucage}

Depuis 1994, Réjean Beaucage est réalisateur à la station CIBL-FM de l'émission AnémixinémA, consacrée à l'actualité des musiques contemporaine, électroacoustique ef actuelle. Le Conseil québécois de la musique lui remettait en février 1998 un prix Opus pour la réalisation de l'émission AnémixinémA, choisie Production médiatique de l'année. Chroniqueur en musique classique à l'hebdomadaire Voir de Montréal, il collabore aussi régulièrement aux revues Esse larts + opinions) et Possibles.

\section{Ned Bouhalassa}

Montréalais né en France, Ned Bouhalassa est un compositeur de musique de concert et de trames sonores pour le petit et le grand écran. Ses œuvres acousmatiques sont enregistrées sur l'étiquette Empreintes DIGITALes. En 2002, il entame une nouvelle pièce d'envergure intitulée mOrpheus, sa première pour son et image. Adresse électronique : ned@nedfx.com

\section{Yves Daoust}

Yves Daoust a étudié au Conservatoire de musique de Montréal, avec Gilles Tremblay /composition ef analyse) et par la suite en France au
GMEB (Groupe de musique expérimentale de Bourges), sous la direction d'Alain Savouret.

Il a travaillé comme concepteur sonore à l'Office national du film du Canada (1976-1979). Depuis 1980, il est professeur au Conservatoire de musique du Québec à Montréal où il a développé un programme d'études en composition électroacoustique.

Très actif sur la scène professionnelle, et l'un des pionniers de la musique électroacoustique au Québec, il a contribué à la fondation et au développement de différents organismes liés à la promotion et à la diffusion de cette musique, dont l'ACREQ, organisme qu'il a dirigé pendant près de dix ans.

Sa production touche à plusieurs facettes du médium : musiques de film, de scène, spectacles pluridisciplinaires, œuvres de concert (musiques électroacoustiques de studio, musiques instrumentales et mixtes, musiques électroacoustiques en direct), créations radiophoniques.

\section{Jean-François Denis}

Jean-François Denis a étudié au Mills College (Oakland, Californie) avec David Rosenboom (maîtrise, 1984). II pratique l'électroacoustique en direct, seul ou en ensemble, et en différé, pour la danse et l'interdisciplinaire, depuis 1982.

II a enseigné l'électroacoustique à I'université Concordia à Montréal (1985-1989) où il a organisé les séries de concerts du Groupe électroacoustique de Concordia (GÉC). $\|$ a été membre fondateur et premier président de la Communauté électroacoustique canadienne (CÉC). En 1994, il reçoit un prix de la ligue canadienne de compositeurs pour son engagement exceptionnel à l'égard des compositeurs canadiens et, en 1995, le prix Jean A. Chatmers de composition musicale, catégorie diffuseurs, pour sa contribution à la diffusion de nouvelles œuvres (électroacoustiques) canadiennes. II est directeur de DIFFUSION i MéDIA (1989) qui produit et distribue les disques Empreintes DIGITALes (1990), SONARt (1992) et No Type (2002).

Jean-François Denis est un des trois cofondateurs de Réseaux (1991) qui produit les séries de concerts Rien à voir à Montréal.

\section{Robert Normandeau}

Détenteur d'une maîtrise et d'un doctorat en composition de l'Université de Montréal, Robert Normandeau est membre fondateur de la Communauté électroacoustique canadienne (CÉCl. directeur artistique et producteur de la série Clair de terre, présentée au Planétarium de Montréal de 1989 à 1993 par l'ACREQ, et cofondateur de Réseaux, qui a notamment produit onze éditions de la série Rien à voir. Lauréat des concours internationaux de Bourges, Phonurgia-Nova, LuigiRussolo, Noroit-léonce Petitot, Stockholm et Ars Electronica /Golden Nica en 1996), il est professeur de composition à l'Université de Montréal depuis 1999. Le Conseil québécois de la musique lui a décerné le prix Opus du compositeur de l'année (1998-1999) et du disque de l'année (1999) pour son CD Figures (Empreintes DIGITALes, IMED 9944). II a remporté également le Masque 2001 de la meilleure musique de théâtre décerné par l'Académie québécoise du théâtre pour Malina. Après avoir réalisé quelques œuvres instrumentales et mixtes, son travail de compositeur est aujourd'hui essentiellement consacré à la musique acousmatique.

\section{Alain Savouret}

Alain Savouret possède une double formation, classique ICNSM de Paris avec Elsa Barraine, Olivier Messiaen, Marcel Beaufill et expérimentale (Service de la recherche de l'ORTF, Pierre Schaeffer). II en découle une 
démarche musicale fondamentalement transversale, passant selon l'époque et les lieux traversés par la composition électroacoustique ou instrumentale, l'improvisation non idiomatique, la réalisation vidéo/son multicanal, quelques écrits ainsi que, comme une sorte de synthèse, la «maîtrise d'œuvre» : action de créa tion sur un long terme en appui sur un "patrimoine spécifique "lles hommes d'un certain lieu à un certain moment, leurs usages, leur environnement, leurs sons...). Cette démarche inventive où le musical est engendré par la relation au contexte favorisera l'émergence de l'«auralité » dans la réflexion théorique sur l'acte musical et sa pratique actuelle.

Il a créé la classe d'improvisation générative au CNSM de Paris, en 1992. Grand Prix des compositeurs de la SACEM en 1982, il est également membre de l'Académie internationale de musique électroacoustique de Bourges.

\section{Alain Thibault}

Directeur artistique de l'ACREQ et membre fondateur de la Communauté électroacoustique canadienne (CÉC), il a participé notamment à la production de pièces de théâtre lavec Gilles Maheu pour Carbone 14), de chorégraphie lavec Edouard Lock pour Lalala Human Steps et Paul-André Fortier) et de vidéos d'art (avec François Girard). Avec l'artiste visuel Yan Breuleux, il a créé la pièce multimédia FausTechnology qui a été présentée lors des événements Elektra 2000 et au International Symposium for Electronic Arts ISEA 2000 à Paris. De plus, leur vidéo-musique a-light a obtenu une mention au prix Ars Electronica 1998 (Linz, Autriche). La musique d'Alain Thibault a été présentée au Canada et à l'étranger, notamment par la Société de musique contemporaine du Québec (SMCQ), par New Music Concerts à Toronto, au Digicon '85, au deuxième congrès d'art et de créativité par ordinateur, à Vancouver, au Festival international de musique expérimentale de Bourges, Synthèse (France) et à Tokyo (Japon).

\section{Barry Truax}

Barry Truax est professeur agrégé au département de communications et au Centre des arts de l'Université Simon Fraser, en Colombie-Britannique, où il enseigne la communication acoustique et la musique électroacoustique. II a reçu une formation scientifique d̀ I'Université Queen's, à Kingston, et une formation musicale à l'Université de la Colombie-Britannique. II a également étudié à l'Institut de sonologie d'Utrecht, en Hollande, avec Koenig et laske.

Il a mis au point et utilisé depuis 1972 le système de composition et de synthèse sonore par ordinateur appelé PODX, qui a fourni le matériau de la plupart de ses compositions, en conjonction avec les techniques classiques et électroniques.

En 1985, il a dirigé la International Computer Music Conference, tenue à Vancouver, où il a lancé son disque Sequence of Earlier Heaven sur sa propre étiquette : Cambridge Street Records. Cinq de ses œuvres figurent sur le disque compact Digital Soundscapes publié par SCR et Wergo.

Son œuvre Riverrun a été primé Magisterium /catégorie ouverte seulement aux compositeurs d'électroacoustique ayant plus de vingt ans d'expériencel au concours international de musique électroacoustique de Bourges en France (1991). En 1999, il reçoit également un prix d'excellence pour son enseignement à l'Université Simon Fraser. 